\title{
Discrepancies in HLA Typing by PCR-SSOP and SBT Techniques: A Case Study
}

\author{
HÉLDER SPÍNOLA, ${ }^{1}$ JÁCOME BRUGES-ARMAS, ${ }^{2}$ AND ANTÓNIO BREHM ${ }^{1}$
}

\begin{abstract}
Six hundred twenty-one samples from Portugal, the Cabo Verde archipelago, and Guinea-Bissau were typed for $H L A-A, H L A-B$, and $H L A-$ $D R B 1$ using the polymerase chain reaction-sequence-specific oligonucleotide probe (PCR-SSOP) method and the sequence-based typing (SBT) method to characterize and compare discrepancies between the two methods. Fifty-three alleles ( $4.27 \%$ of 1,242 chromosomes typed) identified by the PCR-SSOP method were not concordant with the results obtained using the SBT method. Thirty-four (2.74\% of total chromosomes typed) PCR-SSOP mistyping results were discrepancies inside the same allele group and 19 others $(1.53 \%$ of total chromosomes typed) were relative to nonconcordant results between different groups. PCR-SSOP allele mistyping is the result of interpretation difficulties resulting from less intense, absent, or dubious hybridization patterns. Noncommercial PCR-SSOP procedures are highly exigent on the technicians' experience and the availability of properly calibrated high-precision equipment.
\end{abstract}

The human leukocyte antigen (HLA) is one of the most polymorphic genetic systems in humans and consists of a closely linked set of genes. HLA genes are highly important for medical purposes (e.g., transplantation, autoimmune diseases, and allergies) and in the anthropological and forensic fields (Boehncke et al. 1998; Riley and Olerup 1992).

$H L A$ typing has experienced a rapid evolution since DNA techniques appeared 20 years ago, especially because of the introduction of the polymerase chain reaction (PCR) method (Mullis and Faloona 1987). Nowadays, sequencespecific priming (SSP) (Bunce et al. 1995), polymerase chain reaction-sequencespecific oligonucleotide probes (PCR-SSOP) (Middleton 2000), and sequencebased typing (SBT) (Kurz et al. 1999) are the most generalized HLA typing techniques that allow a medium- or high-resolution throughput. The accuracy of these typing techniques is important because organ and tissue transplantation rejection,

\footnotetext{
${ }^{1}$ Human Genetics Laboratory, University of Madeira, Campus of Penteada, 9000-399 Funchal, Portugal.

${ }^{2}$ SEEBMO, Hospital Santo Espírito de Angra do Heroísmo, Terceira, Azores, Portugal; and Institute for Molecular and Cell Biology (IBMC), University of Porto, Porto, Portugal.

Human Biology, October 2007, v. 79, no. 5, pp. 537-543.

Copyright () 2007 Wayne State University Press, Detroit, Michigan 48201-1309
}

KEY WORDS: HLA, POLYMERASE CHAIN REACTION-SEQUENCE-SPECIFIC OLIGONUCLEOTIDE PROBE (PCR-SSOP) TECHNIQUE, SEQUENCE-BASED TYPING (SBT) TECHNIQUE, PORTUGAL, CABO VERDE, GUINEA-BISSAU, AZORES ISLANDS. 
particularly of bone marrow, depends on it. HLA matching for transplantation, if not correctly assigned, could be responsible for graft failures (Cicciarelli 2004). Several comparative studies between serology and DNA techniques have been done in the past years; they have shown large discrepancies, leading to the conclusion that DNA techniques are much more reliable than serological techniques (Mytilineos et al. 1990; Mickelson et al. 1993; Donadi et al. 2000; Ferraz et al. 2002; Borelli et al. 2004). However, DNA typing techniques are not free of errors because they depend on sensitive procedures conducted at specific temperatures, good DNA quality, adequate reagents, and also correct interpretation of the results. Despite the importance of the reliability of different HLA DNA typing methods, few comparative studies have been performed between these techniques (Smith et al. 1997).

In the present study we compared results obtained with PCR-SSOP and SBT typing in samples from the Portugal, Cabo Verde, and Guinea-Bissau populations to identify and characterize discrepancies between these two techniques.

\section{Materials and Methods}

The sample population consisted of 621 healthy unrelated males of different origins (Portugal mainland, $n=145$; Madeira Island, $n=185$; Azores archipelago, $n=102$; Cabo Verde, $n=124$; and Guinea-Bissau, $n=65$ ). Genomic DNA was isolated from blood using a phenol-chloroform procedure. The samples were DNA-typed for $H L A$ class I ( $A$ and $B$ ) and class II (DRBI) antigens using a noncommercial medium-resolution PCR-SSOP method, as previously described (Middleton 2000). The primers for the $H L A-A$ and $H L A-B$ loci give a locus-specific product covering exons 2 and 3, and the primers for $H L A-D R B 1$ give a product from exon 2.

The PCR-amplified product was immobilized on a nylon membrane before hybridization with digoxigenin (DIG)-ddUTP labeled probes. One membrane was prepared for each probe hybridization. To differentiate the alleles, a battery of sequence-specific probes is required; the pattern of reactivity of these probes distinguishes the $H L A$ alleles. The hybridization process was developed at a specific temperature for each probe, and then the membranes were washed to remove the excess nonhybridized probe. The hybridization detection of the labeled probes to a complementary sequence present in the PCR-amplified $H L A$ allele was done by adding an antidigoxigenin antibody conjugated with alkaline phosphatase (ALP). The ALP then used CSPD as its chemiluminescent substrate, and the light emitted was detected by autoradiography.

All subjects were typed for $H L A-A$ and $H L A-B$ using the high-resolution sequence-based method outlined by Kurz et al. (1999) and Pozzi et al. (1999), respectively, with minor modifications. $H L A-D R B 1$ was typed through the exon 2 complete sequence. $H L A-D R B 1$ was amplified and sequenced with the groupspecific primers used by Williams et al. (2004), as described previously (Spínola et al. 2005). 
Comparison of PCR-SSOP and SBT Typing of HLA / 539

Table 1. Discrepancies Between HLA-A, HLA-B, and HLA-DRB1 Typing in 1,242 Chromosomes (621 Samples) Using the PCR-SSOP and SBT Methods

\begin{tabular}{|c|c|c|}
\hline PCR-SSOP Typing & SBT Typing & Number of Discrepancies \\
\hline$A * 23$ & $A * 2901$ & 1 \\
\hline$A * 23$ & $A * 2902$ & 1 \\
\hline$A * 030102$ & $A * 030101$ & 8 \\
\hline$A * 3203$ & $A * 0101$ & 1 \\
\hline$A * 0226$ & $A * 0201$ & 5 \\
\hline$B * 3520 / 28$ & $B * 3502$ & 2 \\
\hline$B * 15$ & $B * 4002$ & 1 \\
\hline$B * 40$ & $B * 5001$ & 1 \\
\hline \multirow[t]{2}{*}{$B * 3520$} & $B * 3503$ & 1 \\
\hline & $B * 3508$ & 1 \\
\hline$B * 5107$ & $B * 5101$ & 10 \\
\hline$B * 0804 / 05$ & $B * 0801$ & 2 \\
\hline \multirow[t]{2}{*}{$B * 3520 / 28$} & $B * 3508$ & 1 \\
\hline & $B * 1401$ & 1 \\
\hline \multirow[t]{2}{*}{$B * 5107$} & $B * 5201$ & 1 \\
\hline & $B * 5101$ & 2 \\
\hline$B * 40$ & $B * 4403$ & 1 \\
\hline$B * 40$ & $B * 4402$ & 1 \\
\hline \multirow[t]{2}{*}{$B * 2708$} & $B * 2705 / 13$ & 1 \\
\hline & $B * 1402$ & 1 \\
\hline$B * 50$ & $B * 49$ & 1 \\
\hline$B * 35$ & $B * 5301$ & 1 \\
\hline$B * 40$ & $B * 5001$ & 1 \\
\hline$B * 3520$ & $B * 3501$ & 2 \\
\hline \multirow[t]{2}{*}{$B * 3520$} & $B * 3534$ & 2 \\
\hline & $B * 3502$ & 1 \\
\hline$D R B 1 * 07$ & $D R B 1 * 1301$ & 1 \\
\hline$D R B 1 * 11$ & $D R B 1 * 1301$ & 1 \\
\hline Total & & 53 \\
\hline
\end{tabular}

DNA fragments amplified by PCR were purified and sequenced using ABI Prism BigDye Terminator Cycle Sequencing Ready Reaction Kits (Applied Biosystems, Foster City, California) in an ABI Prism 310 Genetic Analyzer (Applied Biosystems), according to the manufacturer's instructions. Sequencing was always performed in the forward and reverse directions and was processed using the Matchtools Allele Identification package (Applied Biosystems).

Results obtained from both methods were compared for discrepancies. Probe nomenclature on this manuscript is the same as that used by Middleton (2000).

\section{Results}

The allele typing of 621 samples detected some discrepancies between the PCR-SSOP and SBT results (Tables 1 and 2). Fifty-three alleles (4.27\% of the 
Table 2. Comparison of PCR-SSOP and SBT Results for $H L A-A, H L A-B$, and HLA-DRB1 in 1,242 Chromosomes (621 Samples)

\begin{tabular}{|c|c|c|c|c|c|c|c|c|}
\hline & \multicolumn{2}{|c|}{$H L A-A$} & \multicolumn{2}{|c|}{$H L A-B$} & \multicolumn{2}{|c|}{$H L A-D R B 1$} & \multicolumn{2}{|c|}{ Total } \\
\hline & $N$ & $\%$ & $N$ & $\%$ & $N$ & $\%$ & $N$ & $\%$ \\
\hline No discrepancy & 1,226 & 98.71 & 1,207 & 96.94 & 1,240 & 99.84 & 1,189 & 95.73 \\
\hline Discrepancy & 16 & 1.29 & 35 & 2.82 & 2 & 0.16 & 53 & 4.27 \\
\hline Discrepancy in the same allele group & 13 & 1.05 & 21 & 1.69 & 0 & 0 & 34 & 2.74 \\
\hline Discrepancy in different allele groups & 3 & 0.24 & 14 & 1.13 & 2 & 0.16 & 19 & 1.53 \\
\hline False homozygosity & 0 & 0 & 6 & - & 0 & 0 & 6 & - \\
\hline
\end{tabular}

1,242 chromosomes typed) identified by PCR-SSOP were not concordant with the SBT results. These 53 mistyping results had a different distribution among the three loci studied, with 16 cases for $H L A-A, 35$ for $H L A-B$, and only 2 for HLA$D R B 1$. Thirty-four (2.74\% of the total chromosomes typed) PCR-SSOP mistyped results were discrepancies inside the same allele group, and 19 (1.53\% of the total chromosomes typed) were in different groups. Six of all nonconcordant results revealed false homozygous samples, all of them in the $H L A-B$ locus and showing, by the SBT method, another allele belonging to a different group from that already identified by PCR-SSOP. Only 2 of the 53 discrepant results $(A * 030102 / A * 030101$ and $B * 3520 / B * 3534$ ) were found in the same sample; all other instances of incongruent typing were always found in different samples.

HLA-A Mistyping. Some of the 16 cases of incongruent typing between PCRSSOP and SBT on the HLA-A locus could be explained by weak PCR product and hybridization process sensitivity.

The $A * 23 / A * 2901$ incongruence found in this study in only one sample was the result of a dubious hybridization pattern resulting from a weak PCR amplification. A reanalysis of the PCR-SSOP hybridization pattern of this sample revealed some weak reactions, previously assumed as background, that is, resulting from the $A * 29$ presence (probes $\mathrm{B}, \mathrm{R}$, and $\mathrm{Z}$ ).

Several samples revealed $A * 030102 / A * 030101$ and $A * 0226 / A * 0201$ incongruences between PCR-SSOP and SBT. The PCR-SSOP hybridization patterns between these two pairs of alleles differ only by one probe (A525). For the $A * 030102 / A * 030101$ incongruence this probe reaction was missed as a result of a weak PCR. In the samples in which the incongruence $A * 0226 / A * 0201$ was detected, a deficient washing membrane or an excess exposure time of the membranes to x-ray film could be the cause of the background that was incorrectly interpreted as a positive reaction.

The $A * 3203 / A * 0101$ incongruence between PCR-SSOP and SBT revealed hybridization failure on probes $150 \mathrm{~V}, \mathrm{~A} 26$, and 163R. The weak PCR amplification obtained or an unstable hybridization temperature is assumed to be the cause of these hybridization failures. 
The analyses of the $A * 23 / A * 2902$ incongruence did not reveal any explanation for it. The probe reactions were clear, and the probes that should be assigned in the presence of $A * 29$ were completely negative with no background.

HLA-B Mistyping. $\quad H L A-B$ was the locus where we found the highest prevalence of mistyping results. The incongruences between the PCR-SSOP and SBT methods on $H L A-B$ loci included six false homozygotes. A probe pattern hybridization reanalysis of these false homozygous samples did not reveal any background or weak hybridization results that could be misinterpreted. Because the PCR amplification of the $H L A-B$ locus was difficult and mostly gave weak results in our experiment, we assume that all six false homozygous samples were the consequence of hybridization probe failure.

Other incongruences between the PCR-SSOP and SBT results on the HLA$B$ locus were the consequence of incorrectly interpreted weak positive probe reactions as background. Background in the hybridization pattern, resulting from deficient washing membrane and excess exposure of membranes to x-ray film, was in some cases incorrectly interpreted as a positive reaction.

A false-negative on PCR-SSOP probe BL05, misinterpreted as background, was responsible for 17 PCR-SSOP mistypings on the $H L A-B$ locus. The most prevalent incongruence resulting from this false-negative was $B * 5107 / B * 5101$, which occurred in 10 different samples. This same situation was also responsible for the $B^{*} 3520 / 28 / B^{*} 3502, B^{*} 3520 / B^{*} 3503, B^{*} 0804 / 05 / B^{*} 0801$, and $B^{*} 3520 /$ $B * 3501$ incongruences.

A false-positive on PCR-SSOP probe BL21 was involved, in addition to some false-negative probes, in five different incongruent typings: $B * 40 / B * 4403$, $B * 40 / B * 4402, B * 2708 / B * 2705 / 13, B * 50 / B * 49$, and $B * 35 / B * 5301$. These falsepositives for probe BL21 were the result of an incorrect background interpretation as positive hybridization.

HLA-DRB1 Mistyping. The two samples that revealed incongruent results between the PCR-SSOP and SBT methods at the HLA-DRBI locus $(D R B I * 11$ ) $D R B 1 * 1301$ and $D R B 1 * 07 / D R B 1 * 1301)$ were a consequence of incorrect probe pattern interpretation. Some background resulting from excess exposure to $\mathrm{x}$-ray film or deficient membrane excess probe washing were interpreted as positive probe hybridization (probes 1006, 2810, and DR24 in DRBI*07/DRB1*1301 incongruence; probe 5703 in $D R B 1 * 11 / D R B 1 * 1301$ incongruence), and some weak positive probe reactions were considered background (probes DR86 and DRB13 in $D R B 1 * 11 / D R B 1 * 1301$ incongruence; probe DR86 in DRB1*07/DRB $1 * 1301$ incongruence).

\section{Discussion}

The incongruences found between the PCR-SSOP and SBT results in this study are the result of the high sensitivity of the PCR-SSOP technique. Many of the 


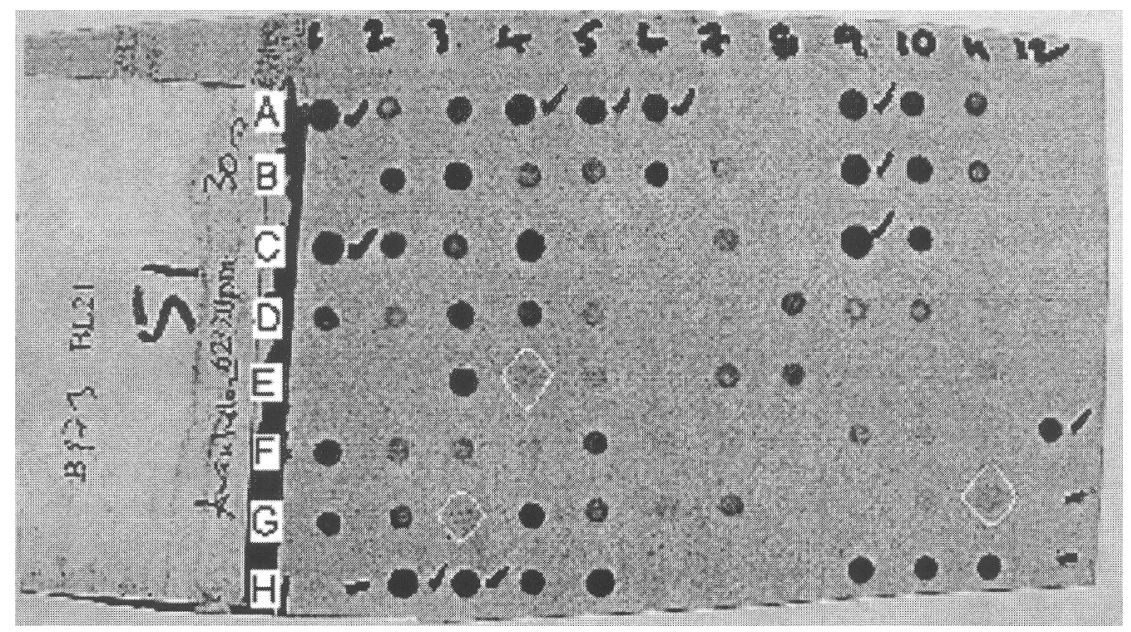

Figure 1. Hybridization pattern of probe BL021 for $H L A-B$ on 96 samples showing some background signals that were misinterpreted as weak positive results. Some examples of interpretation difficulties resulting from dubious hybridization patterns are the following: $3 \mathrm{G}$ dot background interpreted as positive led to mistyping a $B^{*} 44$ allele as $B^{*} 40 ; 4 \mathrm{E}$ dot background interpreted as positive led to mistyping a $B * 49$ allele as $B * 50 ; 11 \mathrm{G}$ dot background interpreted as positive led to mistyping a $B * 44$ allele as $B * 40$.

53 (4.27\%) cases of mistyped HLA alleles using PCR-SSOP-assuming that SBT characterization was the correct one-were the result of interpretation difficulties due to less intense, absent, or dubious hybridization patterns (Figure 1). These problematic hybridization patterns could be explained by poor-quality and lowquantity DNA amplification and the occurrence of some variations at the highly sensitive specific temperatures that probe hybridization needs in the PCR-SSOP method. The washing membrane process is also another sensitive step that, if not completely achieved, could leave behind some background signals that make it difficult to correctly interpret the hybridization pattern. Otherwise, the PCR-SSOP method is covering only certain nucleotide bases, whereas the SBT method covers all nucleotide sequences. In addition, the computer program used to analyze the PCR-SSOP results would not take account of as many alleles as the SBT analysis program does.

Equipment for noncommercial PCR-SSOP needs to be high precision; incorrect results could be obtained if the equipment is not properly calibrated or properly used. This is the case for water baths for high specific temperatures in probe hybridization. For laboratory staff and researchers who undertake the first steps in HLA typing, noncommercial PCR-SSOP is not a recommended technique because it requires a large time and equipment investment until a satisfactory quality level can be achieved. On the contrary, even noncommercial HLA SBT relies on mostly automated steps that are easy to implement and are not sensitive to technique and interpretation errors. 
Acknowledgments This research was supported by the European Community through Program Interreg IIIB Madeira-Açores-Canárias (project MAC/2.3/A6) and by the Portuguese Foundation for Science and Technology (FCT) through Project POCI/BIA-BCM/60440/ 2004.

Received 30 March 2006; revision received 18 May 2007.

\section{Literature Cited}

Boehncke, W. H., C. Loeliger, P. Kuehnl et al. 1998. Identification of HLA-DR and -DQ alleles conferring susceptibility to pollen allergy and pollen associated food allergy. Clin. Exp. Allergy 28(4):434-441.

Borelli, S. D., D. Souza, K. F. F. Freitas et al. 2004. Comparative study between serological and PCRSSP methods to determine class II HLA molecules. Acta Scientiarum Health Sci. Maringá 26(2):337.

Bunce, M., M. Barnardo, M. Browning et al. 1995. Phototyping: Comprehensive DNA typing for HLA$A, B, C, D R B 1, D R B 3, D R B 4, D R B 5$, and $D Q B 1$ by PCR with 144 primer mixes utilizing sequence-specific primers (PCR-SSP). Tissue Antigens 46:355-367.

Cicciarelli, J. C. 2004. HLA typing immunogenetics and transplantation. Curr. Opin. Organ Transplant. 9(1):1-7.

Donadi, E. A., M. Cássia, P. Santos et al. 2000. HLA-DR and HLA-DQ typing: A comparative study using serology and restriction fragment length polymorphism (RFLP) analysis. Medicina Ribeirão Preto 33:27.

Ferraz, A. S., L. S. Saber, J. C. Voltarelli et al. 2002. Comparative study of HLA-DR typing by serology and sequence-specific primer analysis in a genetically highly diverse population of kidney transplant recipients. Transplant. Proc. 34:463-465.

Kurz, B., I. Steiert, G. Heuchert et al. 1999. New high resolution typing strategy for HLA-A locus alleles based on dye terminator sequencing of haplotypic group-specific PCR-amplicons of exon 2 and exon 3. Tissue Antigens 53:81-96.

Mickelson, E., A. Smith, S. McKinney et al. 1993. A comparative study of HLA-DRB1 typing by standard serology and hybridization of nonradioactive sequence-specific oligonucleotide probes to PCR-amplified DNA. Tissue Antigens 41(2):86-93.

Middleton, D. 2000. PCR-SSOP class I and class II (DRB1). In ASHI Laboratory Manual, 4th ed., G. A. Land, R. M. Strothman, and A. B. Hahn, eds. Lenexa, KS: American Society for Histocompatibility and Immunogenetics, 1-23.

Mullis, K. B., and F. Faloona. 1987. Specific synthesis of DNA in vitro via a polymerase catalyzed chain reaction. Meth. Enzymol. 155:335-350.

Mytilineos, J., S. Scherer, and G. Opelz. 1990. Comparison of RFLP-DR beta and serological HLA-DR typing in 1,500 individuals. Transplantation 50(5):870-873.

Pozzi, S., A. Longo, and G. B. Ferrara. 1999. HLA-B locus sequence-based typing. Tissue Antigens 53:275-281.

Riley, E., and O. Olerup. 1992. HLA polymorphism and evolution. Immunol. Today 13:333-335.

Smith, A. G., K. Matsubara, E. Mickelson et al. 1997. A comparative study of HLA-DRB typing by transcription-mediated amplification with the hybridization protection assay (TMA/HPA) versus PCR/SSOP. Hum. Immunol. 55(1):74-84.

Spínola, H., D. Middleton, and A. Brehm. 2005. HLA genes in Portugal inferred from sequence-based typing: In the crossroad between Europe and Africa. Tissue Antigens 66:26-36.

Williams, F., A. Meenagh, R. Single et al. 2004. High resolution HLA-DRB1 identification of a Caucasian population. Hum. Immunol. 65:66-67. 
Copyright of Human Biology is the property of Wayne State University Press and its content may not be copied or emailed to multiple sites or posted to a listserv without the copyright holder's express written permission. However, users may print, download, or email articles for individual use. 